\title{
Acomodo de figuras irregulares en áreas irregulares para el corte de cuero
}

\section{Fitting Irregular Shape Figures into Irregular Shape Areas for the Nesting Problem in the Leather Industry}

\author{
Guevara-Palma Luis \\ Centro de Alta Tecnología \\ Facultad de Ingeniería \\ Universidad Nacional Autónoma de México \\ Correo:luisguevara@comunidad.unam.mx \\ Santillán-Gutiérrez Saúl Daniel \\ Centro de Alta Tecnología \\ Facultad de Ingeniería \\ Universidad Nacional Autónoma de México \\ Correo:saulsan@unam.mx \\ Tang-Yu Xu \\ Centro de Alta Tecnología \\ Facultad de Ingeniería \\ Universidad Nacional Autónoma de México \\ Correo:tang@unam.mx
}

\author{
Dorador González Jesús-Manuel \\ Centro de Diseño y Manufactura \\ Facultad de Ingeniería \\ Universidad Nacional Autónoma de México \\ Correo:dorador@unam.mx \\ Lara-Jiménez Claudia Ivette \\ Centro de Alta Tecnología \\ Facultad de Ingeniería \\ Universidad Nacional Autónoma de México \\ Correo:clausula81@hotmail.com
}

Información del artículo: recibido: marzo de 2013, reevaluado: abril de 2013, aceptado: marzo de 2014

\section{Resumen}

El problema del acomodo de figuras irregulares dentro de áreas irregulares se ha estudiado desde varios enfoques debido a su aplicación en diferentes industrias. El caso particular del corte de cuero implica varias restricciones que hacen difícil la automatización de este problema, pues es necesario cumplirlas para poder generar productos con la calidad requerida por los clientes. El presente documento presenta una metodología para el acomodo de figuras irregulares en un área irregular (piel) considerando las restricciones establecidas por la industria del calzado, así como los resultados de dicha metodología al aplicarlos mediante un sistema de cómputo. El alcance del sistema es desarrollar un algoritmo que pueda integrarse en un prototipo funcional, que opere bajo los lineamientos de una línea de producción de una industria patrocinadora. Pruebas de laboratorio del sistema de cómputo arrojan reducciones del tiempo de $70 \%$ con respecto a sistemas comerciales y una mejora de 5 a $7 \%$ del área que puede lograr utilizar un operario.

\section{Descriptores:}

- corte de cuero

- optimización en acomodo de figuras irregulares

- nesting

- sistema de producción 


\begin{abstract}
The nesting problem of irregular shapes within irregular areas has been studied from several approaches due to their application in different industries. The particular case of cutting leather involves several restrictions that add complexity to this problem, it is necessary to generate products that comply with the quality required by customers This paper presents a methodology for the accommodation of irregular shapes in an irregular area (leather) considering the constraints set by the footwear industry, and the results of this methodology when applied by a computer system. The scope of the system is to develop a working prototype that operates under the guidelines of a commercial production line of a sponsor company. Preliminary results got a reduction of $70 \%$ of processing time and improvement of $5 \%$ to $7 \%$ of the area usage when compared with manual accommodation.
\end{abstract}

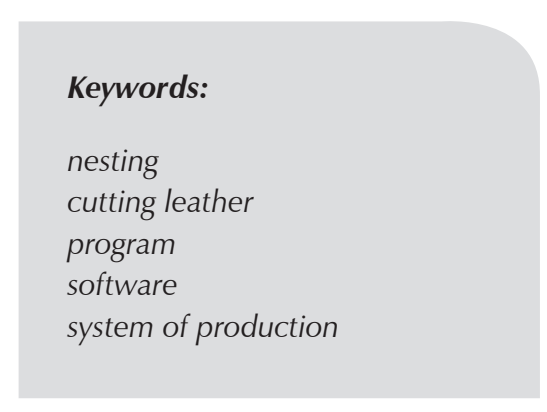

\section{Introducción}

El problema del acomodo de figuras irregulares dentro de otras figuras irregulares se ha estudiado desde diferentes ángulos, debido a su aplicación en diferentes industrias como es el caso del aprovechamiento de la piel, tela, lámina, industria alimenticia y de decoración, donde es importante determinar el mejor arreglo que aproveche al máximo posible la materia prima.

La mayoría de los trabajos desarrollados no han considerado el caso de la irregularidad de la superficie del objeto que recibirá las figuras, (como sería el caso de áreas de diferente espesor y estiramiento en una piel o cambios en el grano de una hoja metálica) o la posibilidad de rotar las figuras a acomodar a un ángulo dado. Para la solución del problema de acomodo, el uso de métodos heurísticos permite mayor generalidad en su desarrollo. Esto permite que la creación de nuevas aplicaciones a partir de dichos métodos sea menos complicada.

Para resolver el problema del acomodo de figuras irregulares se han utilizado diferentes técnicas metaheurísticas como:

- Algoritmos genéticos

- Redes neuronales

- Búsqueda tabú

- Recocido simulado

El problema de estos métodos es compaginar la eficiencia en el acomodo con el tiempo de obtención de la solución, ya que por su naturaleza, buscan obtener el óptimo real y el tiempo se extiende hacia el infinito, por lo que es necesaria su interrupción. Un ejemplo de esto es el trabajo de Crispin (2005) donde el tiempo mínimo obtenido en un acomodo es de $1.4 \mathrm{~h}$ y variando la población se llegaba a $6 \mathrm{~h}$ lo cual, no es aceptable para una línea de producción.
En la industria del calzado, de la tapicería de piel y la peletería en general, una de las tareas más difíciles de automatizar es el acomodo de la piel previo al corte.

Tradicionalmente se ha resuelto con mano de obra especializada, pero este proceso dificulta mantener un alto índice de calidad en los productos, elevar la producción y tener una mayor flexibilidad para absorber cambios en el diseño de los productos.

Para agilizar el proceso existen sistemas comerciales que realizan esta tarea a diferentes grados de automatización, de acuerdo con las necesidades y posibilidades económicas de la empresa.

Como ejemplo de los sistemas comerciales se pueden mencionar los creados por las empresas Taglio y Zipor. Ambos sistemas presentan problemas al considerar las zonas de estiramiento de la piel y pueden ser muy lentos para encontrar un acomodo "óptimo".

Taglio incorpora la opción de trabajo fuera de línea, permitiendo que el proceso de acomodo se realice sin la presión de mandar el resultado al equipo de corte, lo cual hace que el tiempo utilizado para el acomodo sea más largo, y da la posibilidad de realizar varios acomodos simultáneamente en varios equipos y hacer una cola para realizar el corte.

Por otra parte, Zippor realiza un acomodo que considera menos variables, de manera que aunque no sea el óptimo un operario lo puede modificar, con lo cual la efectividad del acomodo queda comprometida.

El presente trabajo se basa en el acomodo de figuras o piezas en la industria del calzado, pero es posible llevar los resultados a otras industrias. Se presenta lo siguiente:

- Un estudio de las reglas que se siguen en el proceso de corte manual para determinar la heurística seguida por los trabajadores para realizar el acomodo.

- A partir de la heurística encontrada se definió una metodología para llevar a cabo el acomodo de figu- 
ras irregulares en una piel, considerando las restricciones y métodos de trabajo establecidas en la industria del calzado.

- Un sistema de cómputo prototipo que realiza el acomodo de figuras irregulares en una piel digitalizada basado en la metodología presentada.

\section{Corte de cuero manual}

El problema de fondo es el acomodo de piezas irregulares en un área irregular. El caso de estudio que se presenta utiliza cuero, pero se pueden considerar otros escenarios para que el software se pueda aplicar en otras industrias que manejan diferentes materias primas como hojas, corteza, telas, etcétera. El caso de la industria del calzado es muy completo, ya que involucra varias restricciones, que no se encuentran juntas en algún otro caso.

\section{Características de la piel}

En primer término se presentan las características de la piel o cuero, que funcionará como figura irregular en la que se acomodarán las piezas o figuras irregulares.

- Irregularidad externa. El contorno de la piel es diferente en cada caso.

- Zonas de calidad. La piel se divide en zonas de calidad (máximo 3) basadas en la combinación de la textura y espesor. El tamaño y forma de las zonas varían en cada piel (figura 1).

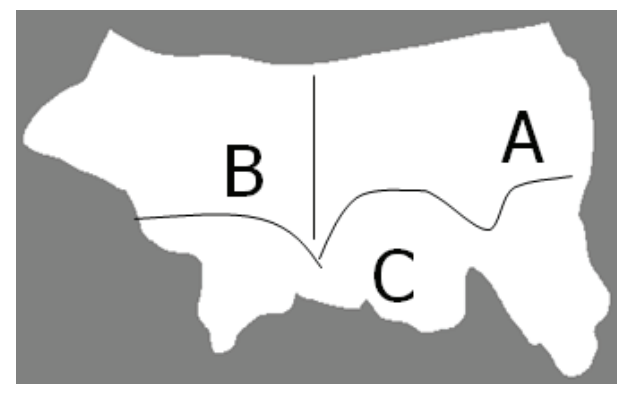

Figura 1. Zonas de calidad

- Zonas de estiramiento. Son zonas en las cuales la piel se estira más fácilmente hacia una dirección. Las figuras que se acomodarán en estas zonas se ven afectadas por el estiramiento de su piel para su funcionamiento (figura 2).

- Defectos. Los defectos que existen en las pieles como son estrías, raspones, piquetes de insectos o manchas, se marcarán y afectará a una zona de la piel.

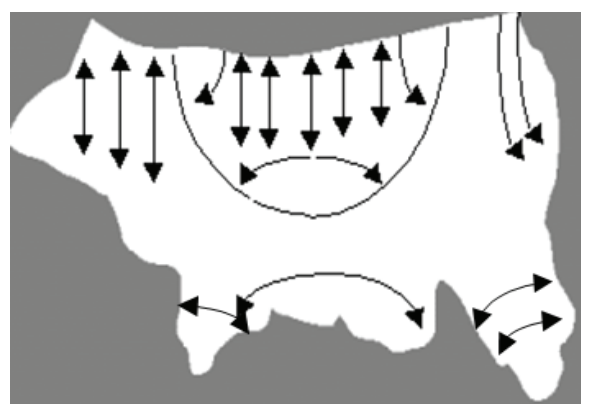

Figura 2. Zonas de estiramiento

Características de las figuras

A continuación se presentan las características de las figuras por acomodar:

- Zona de calidad. Cada figura por su funcionamiento tiene asignada una zona de calidad donde debe ubicarse en la piel. Una figura puede ubicarse en una zona de calidad de mayor nivel al especificado, pero no en una de menor nivel.

- Contorno de la figura. El contorno es diferente en cada figura, es posible que el contorno de una figura sea similar, pero puede variar el tamaño o la orientación (figuras izquierdas).

- Estiramiento. Dependiendo del funcionamiento previsto para cada figura, esta se puede estirar en cierta dirección, la cual se debe alinear a la figura con respecto a un eje paralelo y a la línea de estiramiento de la piel. En algunos casos, se podrá tener una tolerancia de hasta 10 grados con respecto al eje de estiramiento para acomodarlas.

- Defectos. Las figuras no pueden contener áreas con defectos.

\section{Acomodo manual}

En el proceso manual, inicialmente se realiza un proceso de inspección marcado con crayón para determinar los defectos como piquetes, hoyos, raspaduras o estrías, los cuales se consideran de manera diferente dependiendo del producto que se esté fabricando con la piel.

Posteriormente los operarios o cortadores determinan cuáles son las características de cada zona de la piel al hacer nuevas inspecciones visuales y estirar la piel con sus manos en diferentes direcciones para observar su comportamiento y grosor. Una vez que el operario ha determinado las características del área de piel que va a utilizar, elige cuál es la pieza a cortar más adecuada, así como la posición en la que debe ubicarse para cumplir con los requerimientos de la zona de calidad 
adecuada, la alineación del ángulo de estiramiento de la pieza y la piel, así como el manejo de defectos, además de buscar el mejor aprovechamiento de la piel. Acto seguido se procede al corte de la pieza.

Para cortar las piezas de un modelo de cierta talla, se cuenta con un suaje para cada una de las piezas. Para realizar el corte de la pieza, una prensa hidráulica presiona el suaje contra la piel hasta que se obtiene la pieza. Cabe mencionar que los suajes están afilados por ambos lados, ya que para obtener las piezas del zapato "izquierdo" se voltea el suaje y se realiza la misma operación. El proceso de corte manual se ha documentado en el documento Tecnología del Departamento de Corte elaborado por CIATEC (2008).

\section{Análisis del proceso de corte}

$\mathrm{Al}$ analizar las características del proceso de corte se llegó a la conclusión de que el problema a resolver se puede catalogar como un sistema complejo, ya que la materia prima no es lineal en su comportamiento por las siguientes razones:

- La forma del contorno de la piel es variable.

- Las zonas de estiramiento se tienen que reconocer para cada piel.

- La distribución, el tamaño y la forma de las zonas de calidad varía en cada piel.

- Los defectos varían en tipo, cantidad y posición en cada piel.

Al tomar en cuenta las restricciones y características de cada piel, así como las restricciones y características de las piezas que conforman el modelo a producir -descritas en el apartado características de las figurasse generan espacios de solución de millones de combinaciones.

Además, la forma de las figuras cambia en cada producto y cada producto a su vez tiene varios modelos, en algunos casos con la misma geometría, pero en diferentes tamaños.

Un sistema complejo entre otras características tiene un comportamiento que no es lineal, es difícil de modelar con sistemas lineales y, por lo tanto, tiene un alto grado de incertidumbre en su comportamiento.

Un sistema complejo está formado en su mayoría por elementos similares, los cuales al interactuar localmente generan un comportamiento emergente que no es posible explicar al analizar individualmente sus interacciones y es imposible determinar cómo va a desarrollarse más allá de un corto plazo, este tipo de sistemas casi nunca son objeto de procesos de optimización que obtengan un óptimo global. Esto es inherente a la dificultad de modelarlos.

Al utilizar sistemas complejos es necesario probar las partes individuales y una vez funcionando conjuntarlas para que se dé el comportamiento superior. Cada parte se debe afinar en su funcionamiento individual y las desviaciones se deben ir compensando o absorbiendo en cada etapa.

En nuestro caso, el acomodo realizado en cada piel es diferente, ya que involucra conjuntos de figuras que varían mucho en forma y restricciones para poder acomodarlas. Esto hace que el acomodo final en la piel pueda tener más de una solución. El resultado siempre varía al depender de las restricciones al inicio del acomodo. Ya sea por las características de las figuras a acomodar -geometrías, tamaños, etcétera- las características propias de la piel, como su propia geometría, la forma y tamaño de sus zonas de calidad y estiramiento, así como los defectos existentes; al analizar estas características del proceso se llegó a la imposibilidad de determinar con antelación cuál será el resultado del acomodo final en cada caso y el grado de optimización que se obtendría.

Por esta razón, se buscaron diferentes herramientas para atacar el problema, como algoritmos genéticos y agentes inteligentes. Al tratar de caracterizar las herramientas de inteligencia artificial exploradas se encontró la necesidad de determinar con mayor precisión la forma en que estos tenían que actuar, es decir, determinar el algoritmo de acomodo que se comportara de manera consistente en todos los casos. Esto nos llevó a la conclusión de que el problema era difícil de modelar para varias de las herramientas de inteligencia artificial y para obtener los resultados deseados en cuanto al aprovechamiento de la piel y tiempo de proceso.

$\mathrm{Al}$ reconocer esta necesidad, se optó por buscar una heurística de acomodo que tuviera un comportamiento consistente al acomodar las figuras. En el caso del acomodo manual, los operarios no pueden verbalizar este comportamiento, porque implica la toma de varias decisiones simultáneas por cada figura. Se hicieron varias entrevistas en campo y se filmó a los operarios en acción. Un aspecto también difícil de verbalizar y sintetizar fue el proceso de inspección visual de la piel.

$\mathrm{Al}$ analizar la forma de trabajar de los operarios se llegó a la conclusión de que no hacen una optimización global durante el acomodo, sino que desarrollan un acomodo secuencial guiado por varias premisas, que llevan a una suma de optimizaciones parciales.

Se tomó en cuenta que al desarrollar los algoritmos de acomodo, se debe expresar en código de máquina las decisiones del operario, por lo que hay que racio- 
nalizar las reglas y su secuencia para cada etapa del acomodo. Para esto, se definió una estrategia de trabajo que marca el camino para llegar a los resultados esperados.

Dado lo anterior, se diseñó el sistema utilizando las premisas de sistematizar las operaciones de acomodo y anidarlas, buscando la aparición del comportamiento emergente mediante la emulación de las operaciones que sintetizan los operarios al acomodar de una manera secuencial.

\section{Consideraciones para el acomodo}

\section{Consideraciones para el sistema de acomodo}

La metodología del proceso de acomodo se creó siguiendo las reglas heurísticas que se determinaron mediante el estudio de la forma de trabajo de los trabajadores, simulaciones con personal experimentado y entrevistas. Estas reglas son:

- Se acomoda figura por figura.

- Se toman decisiones acerca de cuál figura se va a colocar considerando la sección de la piel donde se realiza el proceso de acomodo en ese momento. Ahí se considera la orientación y ubicación de la figura a acomodar.

- La forma de acomodo se hace siguiendo las reglas del proceso manual de corte (CIATEC, 2008), es decir, de la cola hacia la cabeza y del lomo hacia las patas (figura 3).

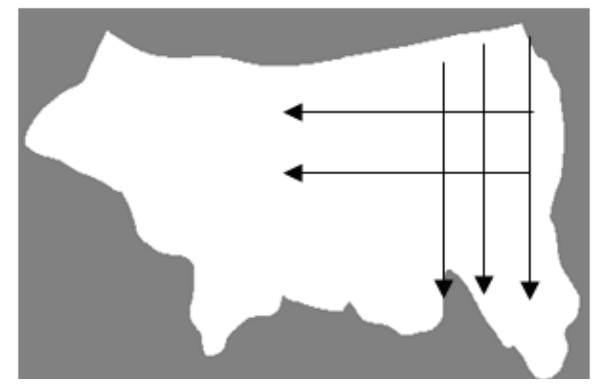

Figura 3. Forma de acomodo

- La piel se divide en zonas rectangulares para disminuir el efecto de las irregularidades del contorno.

- En caso de que una figura salga de la zona de calidad correspondiente, se considera su posición en esa zona, siempre y cuando no pase de $50 \%$ de su área lo que queda afuera. En caso contrario se cambia por una figura de la otra zona de calidad.

- En el caso del estiramiento se tendrá una tolerancia de hasta $10^{\circ}$ para la ubicación de la figura en relación con el ángulo de estiramiento que le corresponda por la zona de la piel en que se encuentra ubicada.

\section{Concepto de óptimo local}

Adicionalmente a las reglas que siguen los operarios, el sistema de acomodo se basa en el principio de que a partir de encontrar la mejor posición o acomodo individual, se llegará al mejor acomodo global. Este concepto se aplica en diferentes niveles durante el proceso de acomodo.

a) Al seleccionar las pieles: para determinar la cantidad de piel necesaria para surtir un pedido, la suma del acomodo de cada piel seleccionada da como resultado el mejor acomodo posible de todo el pedido.

b) A nivel piel: cada una se divide en pequeñas áreas que se denominan: "Marco" (frame), para homogeneizar el acomodo. Por eso se parte de la premisa de que al obtener el mejor acomodo en cada marco se obtiene el mejor acomodo de toda la piel.

c) A nivel de marco: al acomodar pieza por pieza y obtener el mejor acomodo para cada pieza se presume obtener el mejor uso de la piel en el marco correspondiente.

\section{Metodología del sistema de acomodo}

Considerando los principios establecidos, el sistema de acomodo se divide en cuatro etapas:

- Selección de la piel a utilizar

- División de la piel en marcos

- Acomodo grueso

- Acomodo fino

\section{Selección de la piel a utilizar}

Una vez definida la orden de producción, se selecciona un conjunto de pieles con la cantidad de piel necesaria para realizar las piezas. Para esto se considera la cantidad de piel por cada zona de calidad de las mismas, determinada por las partes a acomodar en cada zona, así como la distribución de los diferentes tipos de piel existentes en el almacén. El costo de producción queda severamente afectado por la cantidad de pieles seleccionadas de cada tipo (tabla 1 ).

\section{División de la piel}

Una de las características de la piel es que su contorno no es lineal, varía de una piel a otra y en caso de tener defectos graves, la zona afectada puede cortarse, por 
Tabla 1. Clasificación de las pieles por su porcentaje de aprovechamiento

\begin{tabular}{cc}
\hline TIPO & \%APROVECHAMIENTO \\
\hline A & 98 \\
B & 95 \\
C & 92 \\
D & 90 \\
E & 86 \\
E $^{*}$ & 75 \\
\hline
\end{tabular}

ello la base inicial para el acomodo es el lomo de la piel. $\mathrm{Si}$ tiene un perfil sinuoso, las curvas se proyectarán hacia la parte inferior afectando la siguiente línea de figuras, provocando que disminuya la eficiencia del acomodo (figura 4).

Para evitar este problema se divide la piel considerando las partes del lomo menos sinuosas y abarcado hacia abajo la zona de primera calidad, siguiendo las directrices del acomodo manual, es decir, el principio de hacerlo del lomo a las patas y de la cola a la cabeza (arriba hacia abajo y de derecha a izquierda) (CIATEC, 2008).

Con esta base se genera un segundo renglón de marcos del mismo ancho como el primer renglón, abarcando hasta la parte final de la piel (figura 5).

\section{Acomodo grueso}

$\mathrm{El}$ acomodo grueso es un proceso que permite obtener un primer posicionamiento de la pieza a acomodar sobre la piel y se basa en el llamado "bounding box" de la

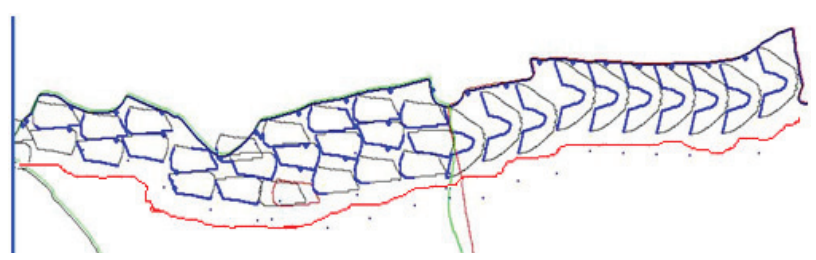

Figura 4. Proyección del contorno en el acomodo de las piezas

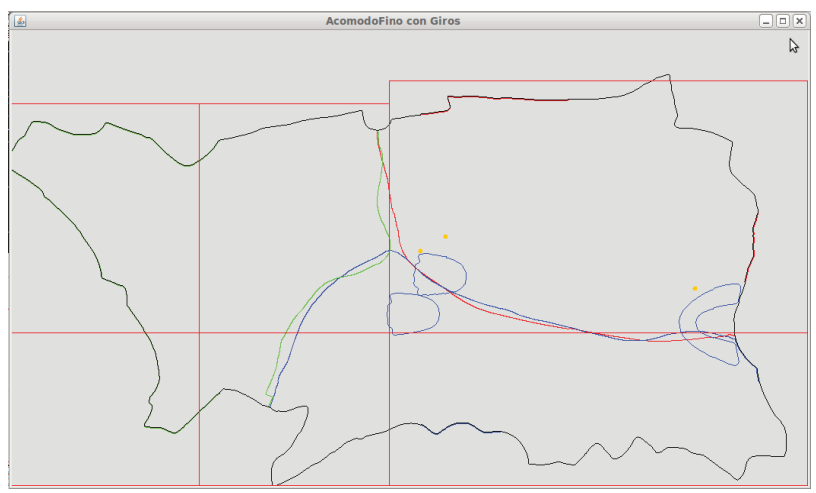

Figura 5. División de la piel en marcos figura, es decir, un rectángulo construido a partir de los puntos extremos de la figura a ubicar para determinar su cercanía, ya sea con piezas previamente acomodadas o con los bordes de la piel (figura 6).

Bounsaythip y Maouche (1997), así como Batchelor (1991) utilizaron el "bounding box" para acomodar las figuras irregulares con algoritmos diseñados para figuras regulares dentro de objetos regulares.

El algoritmo del acomodo grueso consiste en:

a) Ubicar el "bounding box" correspondiente a la figura por ubicar en el límite inferior izquierdo de la figura anterior o el borde de la piel.

b) Mover la figura hacia arriba para alinearse, ya sea con el borde de la piel o la figura que se encuentre en la parte superior (figura 7).

c) Se verifica para determinar si la figura se encuentra dentro de la piel y del marco correspondiente,

c1) si la figura se encuentra dentro de la piel se procede con el acomodo fino;

c2) si la figura está fuera de la piel se considera a la pieza como una figura "virtual". Esta figura virtual se almacena dentro de la estructura de datos que lleva el control de las piezas acomodadas. Estas figuras virtuales son importantes porque se toman como referencia para el acomodo de figuras posteriores, pero al marcar se como virtual se considera que es posible acomodar sobre ellas otras piezas.

\section{Problemas del acomodo grueso}

El "bounding box" aparenta tener buenos resultados, sin embargo, debido a que existen piezas cóncavas, hay casos en que se forma desperdicio de espacio entre las figuras; ya que al delimitar una frontera con los puntos externos, no se permite que la nueva figura ocupe todo el espacio disponible entre ella y la figura ya acomodada (figuras 8 y 9 ).

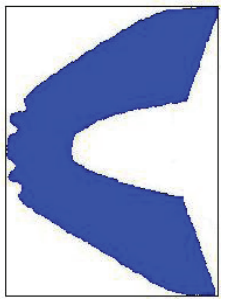

Figura 6. "Bounding box"

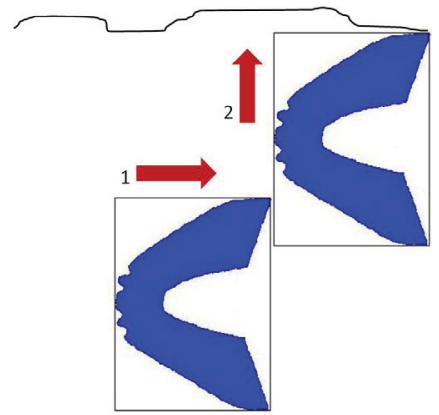

Figura 7. Acomodo grueso. Movimiento de la pieza 


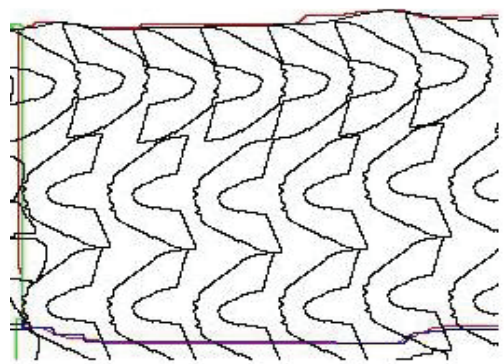

Figura 8. Desperdicio de espacio en acomodo grueso

Para disminuir el desperdicio es necesario conside-

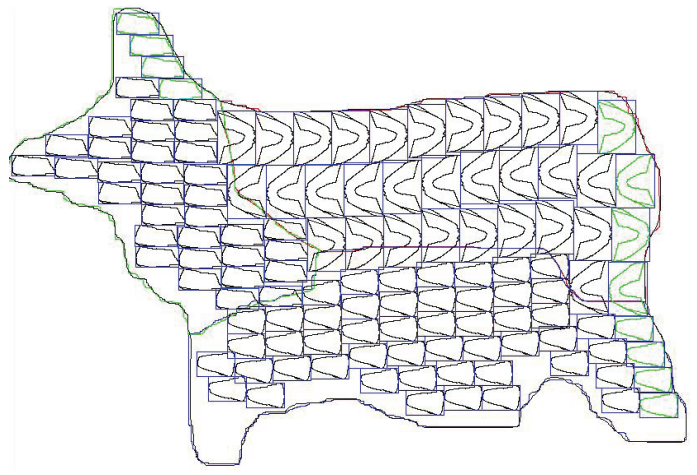

Figura 9. Acomodo grueso utilizando "bounding box"

rar el contorno de las piezas y una aproximación, para ello se manejan como polígonos en lugar de usar cuadriláteros para su acomodo.

\section{Acomodo fino}

El acomodo fino consiste en buscar dentro de un área determinada aquella posición en la cual el desperdicio de espacio es mínimo. El proceso inicia a partir de la posición de la pieza obtenida por el acomodo grueso.

El área de acomodo o "parche" se forma a partir de la frontera derecha y superior que encuentra la figura. Estas fronteras se pueden formar por figuras colocadas anteriormente, por el borde de la piel o ambos. Se determinaron ocho diferentes casos de parches dependiendo de la posición y el entorno de la pieza a colocar. Para determinar cada caso se genera una serie de "sensores", a partir del estado en que se encuentren se determina el tipo de parche a utilizar (figura 10).

Conociendo el tipo de área a generar se toman las fronteras correspondientes (piezas anteriores, orilla de la piel, etcétera) y con base en las medidas de la figura, se calculan los puntos adicionales para formar un área cerrada. El área se genera considerando aproximadamente 1.5 veces el ancho y el largo del "bounding box" de la figura (figura 11).
Una vez generada el área de acomodo la figura se mueve dentro de ella buscando de forma exhaustiva la mejor posición. Esta operación se lleva a cabo en los siguientes pasos:

a) En primer término se determina el ángulo de la línea de estiramiento existente dentro del área de exploración o "parche".

b) Una vez determinado dicho ángulo, se gira la pieza para que coincida la línea de estiramiento de la pieza con la existente en el parche.

c) Una vez que la pieza se encuentra en la posición deseada se realiza un barrido del área de exploración, el cual se lleva a cabo en dos partes.

c1) En la primera parte se barre el espacio del área de exploración moviendo la pieza desde la parte inferior hacia arriba hasta que la pieza toque el borde del área de exploración. La posición que toma la pieza al llegar al borde del área de exploración se considera válida si la pieza se encuentra dentro de la misma y dentro de la piel, y no coincide con alguna falla. Si la posición es válida se calcula el nivel de aprovechamiento de espacio y se almacena el resultado.

Posteriormente se lleva la pieza al borde inferior del área de exploración, avanzando en dirección horizontal repitiendo la operación hasta que la pieza salga de ella (figura 12).

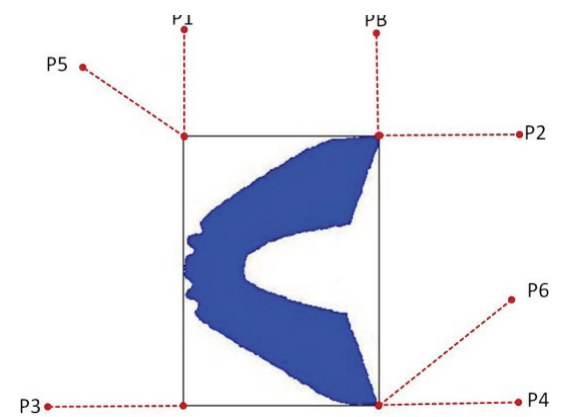

Figura 10. Sensores de posición

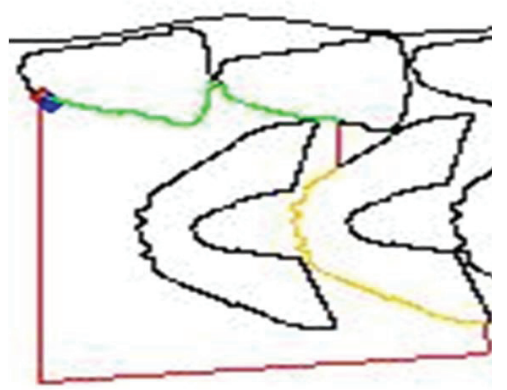

Figura 11. Ejemplo de área de exploración o parche 


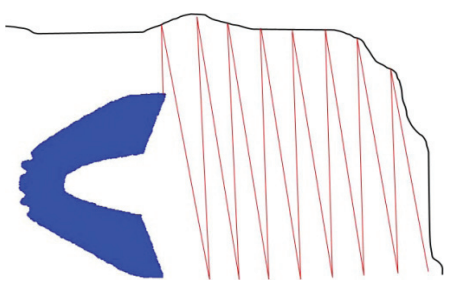

Figura 12. Barrido de vertical para el acomodo fino

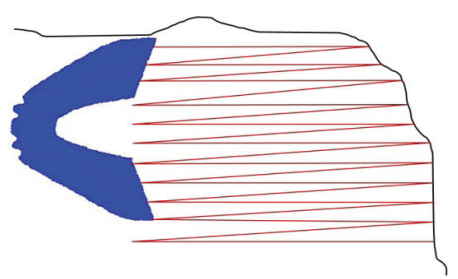

Figura 13. Barrido horizontal para el acomodo fino

c2) La segunda parte del proceso se realiza de forma similar, pero el recorrido seforma horizontal por la parte superior hacia la inferior del área de exploración. En este caso las posiciones validas se consideran cuando la pieza toca el extremo derecho del área de exploración y queda dentro de ella (figura 13).

d) Al terminar de recorrer el área de exploración se hace un análisis de las posiciones válidas que se encontraron, considerando elementos como el área de la figura a colocar, el área del espacio disponible, el espacio ocupado, el espacio no ocupado o desperdiciado, etcétera y se determina cuál fue la posición en la que se generó menor desperdicio, con la pieza en el ángulo especificado al inicio del barrido.

Como es aceptable tener variaciones en la alineación de la pieza con respecto a la línea de estiramiento de $-10^{\circ}$ a $10^{\circ}$, se ajusta la posición de la pieza en incrementos de $5^{\circ}$, posteriormente se gira la pieza a $180^{\circ}$ y se repiten las variaciones especificadas (figura 14). En cada una de las variaciones se repite el barrido recolectándose las mejores posiciones de la pieza. Una vez recolectadas las mejores posiciones de cada ángulo, de este conjunto de posiciones se selecciona aquella cuyo desperdicio de espacio sea menor y se almacena como la posición definitiva.

Ya ubicada la figura se verifica en qué porcentaje se encuentra dentro de la zona de calidad a la que pertenece y si está dentro de la piel. Si no cumple con el criterio de acomodo, se determina cuál es la zona de calidad que tiene más porcentaje de área y se busca en la base de datos una figura de esa calidad, con la cual, se repita el proceso, pero ahora para una pieza nueva (figura 15).

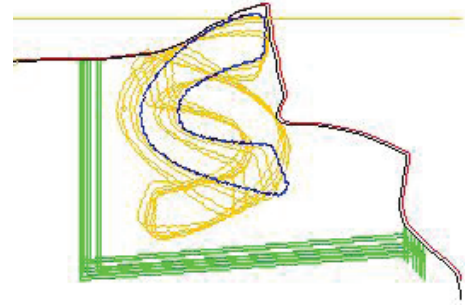

Figura 14. Acomodo de la pieza en diferentes ángulos

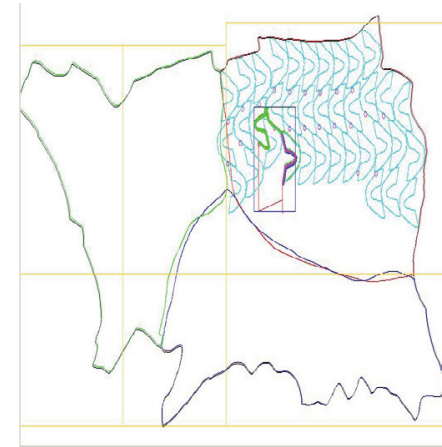

Figura 15. Acomodo fino

\section{Desarrollo}

El sistema se desarrolló con el lenguaje de programación Java debido a la facilidad que brinda para migrar la aplicación a diferentes plataformas de manera transparente.

El sistema se desarrolló modularmente entre varios participantes y para ello se utilizaron plataformas Windows y Linux.

La integración del sistema y sus pruebas se llevaron a cabo en un equipo con un procesador Xeon con $2 \mathrm{~Gb}$ de RAM utilizando sistema operativo Linux.

Para migrar la imagen de la piel ya con las piezas integradas se genera un archivo en formato JPG, el cual se puede importar al sistema de corte existente en la empresa patrocinadora.

\section{Resultados}

La metodología de acomodo en dos etapas presenta buenos resultados, ya que la cantidad de piezas acomodadas se incrementa al usar en combinación los acomodos grueso y fino aprovechando mejor el material, como se muestra a continuación.

En la figura 16 se puede observar el resultado del acomodo grueso en una piel de prueba y en la figura 17 el resultado del acomodo al utilizar los acomodos grueso y fino 


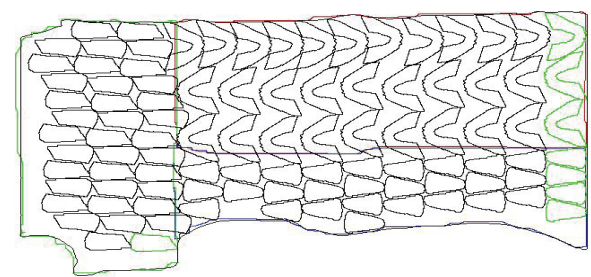

Figura 16. Acomodo grueso

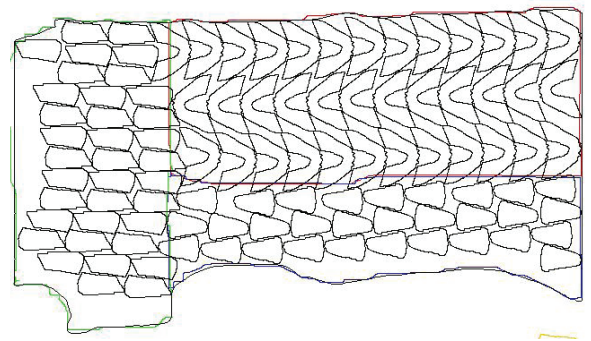

Figura 17. Acomodo fino concluido

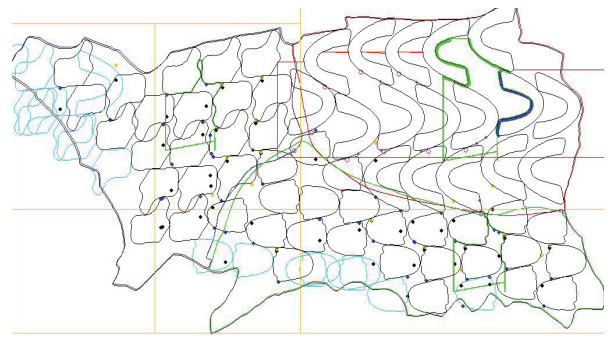

Figura 18. Acomodo de una piel real con figuras reales

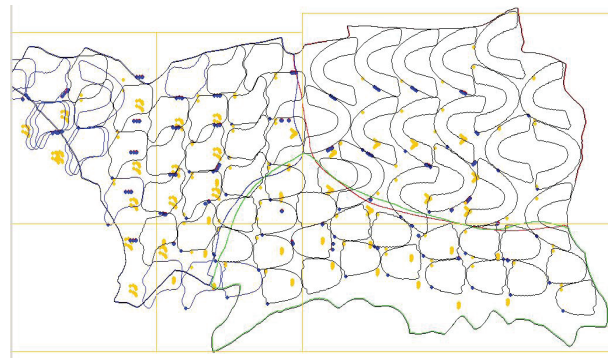

Figura 19. Acomodo considerando giros para las zonas de estiramiento

Tabla 2. Comparación acomodo grueso y fino

\begin{tabular}{cccc}
\hline \multicolumn{2}{c}{ Acomodo grueso } & \multicolumn{2}{c}{ Acomodo fino } \\
\hline $\begin{array}{c}\text { Zona de } \\
\text { calidad }\end{array}$ & Figuras & Zona de & Figuras \\
1 & 33 & 1 & calidad \\
2 & 37 & 2 & 37 \\
3 & 31 & 3 & 36 \\
Total & 111 & Total & 115 \\
\hline
\end{tabular}

Para la industria, la zona de piel de mayor valor es la de calidad 1, por lo que es deseable aprovecharla al máximo acomodando el mayor número de piezas posible.

En pieles reales los resultados son alentadores, ya que el manejo del área de exploración permite ubicar las piezas de forma adecuada, como se muestra en la figura 18.

La comparación de los resultados se muestra en la tabla 2.

La introducción del manejo de los ángulos de estiramiento permite que se aprovechen espacios sin aprovechar, como se observa en la figura 19.

\section{Conclusiones}

El presente trabajo se deriva de un proyecto patrocinado industrialmente; en este proyecto, se realiza desarrollo tecnológico e investigación multidisciplinaria.

Este trabajo en particular reporta el desarrollo de la solución para el problema de acomodo, el cual se ha realizado de acuerdo con las especificaciones que permiten su aplicación en la línea de producción del patrocinador del proyecto.

Se aplicó metodología de diseño para software en este caso específico, sin embargo, el proyecto global siguió la metodología de Pugh (1995).

Hasta el momento no se han encontrado sistemas precedentes que manejen la complejidad de la información del presente trabajo. Los sistemas reportados simplifican demasiado el problema y ofrecen soluciones parciales o no consideran todas las restricciones necesarias.

Los algoritmos desarrollados y la estructura del sistema admiten la integración posterior de herramientas de inteligencia artificial que permiten mayor flexibilidad y eficiencia al sistema.

La estructura del sistema de acomodo permitió integrarlo a un proyecto mecatrónico complejo integrado por sistemas con diferente naturaleza, incluyendo desarrollo de software, un sistema mecánico, reconocimiento de imágenes e interfaces con diferentes sistemas de información.

Hasta el momento, se ha logrado que el sistema sea consistente para los diferentes casos de acomodo, combinando figuras y pieles. La heurística desarrollada está cumpliendo con lo esperado, aunque cabe señalar que faltan aspectos por cubrir.

El rendimiento del sistema y el tiempo en que se realiza el acomodo, permite que sea factible su integración en la línea de producción de fabricantes de zapatos, vestiduras de autos, ropa y similares.

El manejo de los defectos se encuentra en etapa de pruebas y, debido a la estructura del sistema y a la uniformidad en la caracterización de los diferentes elementos (piel, figuras, zonas de calidad, etcétera) la integración del manejo de los defectos ha sido exitosa.

Por otra parte, es importante realizar pruebas con diferentes figuras y pieles para corroborar que el siste- 
ma obtiene porcentajes de utilización de piel consistentes a pesar de las diferentes variables consideradas.

\section{Referencias}

CIATEC. Tecnología del departamento de corte, 2008.

Crispin A., Clay P., Taylor G. Genetic Algorithm Coding Methods for Leather Nesting, Applied Intelligence 23, Springer Science + Business Media, Inc. 2005, pp. 9-20.

Batchelor B.G. Intelligent Image Processing in Prolog, Springer-Verlag, Londres, 1991.

Bounsaythip C. y Maouche S. Irregular Shape Nesting and Placing with Evolutionary Approach, en: Proceedings of the World Manufacturing Congress, Durham, UK, 1997, pp. 27-30.

Pugh S. y Hollins D. Product Design, Newness Butterwoth, Inglaterra, 1995.

Taglio. Sistema CutVision [fecha de consulta: 28 de febrero 2014] [en línea]. Disponible en: <http://www.taglio.it/cutvision_eng/ prodotti/1530.htm>
Zipor, Equipamentos e Tecnologia. Products and Services: Footwear [fecha de consulta: 28 de febrero 2014] [en línea]. Disponible en: http://demo.paloalto.pt/cgibin/eloja21.exe?myid= ziporen\&sh=/ziporen/product/list.htm\&family=0\&mn= footwear.

\section{Este artículo se cita: \\ Citación estilo Chicago}

Guevara-Palma, Luis, Saúl Daniel Santillán-Gutiérrez, Xu Tang-Yu, Jesús Manuel Dorador-González, Claudia Ivette Lara-Jiménez. Acomodo de figuras irregulares en áreas irregulares para el corte de cuero. Ingeniería Investigación y Tecnología, XVI, 01 (2015): 25-34.

\section{Citación estilo ISO 690}

Guevara-Palma L., Santillán-Gutiérrez S.D., Tang-Yu X., DoradorGonzález J.M., Lara-Jiménez C.I. Acomodo de figuras irregulares en áreas irregulares para el corte de cuero. Ingeniería Investigación y Tecnología, volumen XVI (número 1), enero-marzo 2015: 25-34.

\section{Semblanzas de los autores}

Luis Guevara-Palma. Ingeniería en computación por Facultad de Ingeniería, UNAM y maestro en ingeniería (área de planeación). Realiza estudios de doctorado en ingeniería (diseño mecánico) en la División de Estudios de Posgrado, Facultad de Ingeniería, UNAM. Diplomado en bases de datos y diplomado universitario de actualización y formación docente; responsable del área de cómputo del Centro de Diseño y Manufactura, socio de SOMIM (Sociedad de Ingeniería Mecánica). Ha participado en tres proyectos en el CDM y uno en el Centro de Alta Tecnología, Facultad de Ingeniería. Tiene 3 publicaciones y ha dirigido 2 tesis de licenciatura.

Saúl Daniel Santillán-Gutierrez. Profesor titular C, Facultad de Ingeniería UNAM, jefe del Centro de Alta Tecnología, campus Juriquilla, Querétaro. Es líder de 15 proyectos de vinculación industrial e investigación aplicada y responsable académico de las áreas de ingeniería espacial y automotriz en la UNAM, así como del proyecto CONDOR UNAM MAI, (Rusia,Mexico y Taiwan) y QUETZAL MIT UNAM. Cuenta con una patente, 10 registros de autor y 8 publicaciones. Sus líneas de trabajo son aplicaciones de inteligencia artificial en diseño, desarrollo de metodologías de diseño, ingeniería espacial, ingeniería automotriz. Miembro del SNI área Tecnológica, miembro de la Academia de Ingeniería, socio fundador de la SOMIM, SOMECYTA (Sociedad Mexicana de Ciencia y Tecnología Espacial), miembro de SAE, AIUME y otras asociaciones profesionales. Responsable de la REDCYTE (Red Temática CONACYT de la Ciencia y Tecnología del Espacio).

$Y u$ Tang-Xu. Doctor en ingeniería por la Facultad de Ingeniería, UNAM (1988), profesor titular C desde 1998 en la misma faclutad. Estancia sabática en la Universidad de California en Berkeley (1996) y en el Instituto Mexicano del Petróleo (2003). Premios y distinciones: Reconocimiento Catedrático UNAM (1997), Premio Distinción Universidad Nacional para Jóvenes Académicos (1995) y Premio Weizmann (1991). Miembro del SNI (Nivel 2), de la Academia Mexicana de Ciencias, de la Asociación Mexicana de Control Automática y del IEEE. Intereses académicos: desarrollo de sistemas de control adaptable, mecatrónica y aplicaciones en problemas biomédicas e industriales.

Jesús Manuel Dorador-González. Ingeniero mecánico y eléctricista, área mecánica, por la Facultad de Ingeniería, UNAM. Diplomado en automatización industrial y maestría en ingeniería mecánica con la opción de diseño y manufactura en la División de Estudios de Posgrado de la misma facultad, obtuvo el doctorado en Loughborough University, Inglaterra. Ha publicado más de 50 artículos en memorias de congresos nacionales e internacionales y artículos en revistas arbitradas internacionales. Ha dirigido 103 tesis en las áreas de ingeniería mecánica, industrial, mecatrónica y en computación, a nivel licenciatura y posgrado.

Claudia Ivette Lara-Jiménez. Ingeniero industrial por la Facultad de Ingeniería, UNAM (2004), realiza los estudios de maestría en ingeniería de sistemas en la en la misma facultad. Becaria de la Fundación Canada-MexicoEstados Unidos para la Ciencia (FUMEC) en 2004. Participa en el CDM en un proyecto conjunto de identificación de oportunidades de la industria electrónica en el área automotriz. Participa en un proyecto para la industria del calzado en conjunto con el Centro de Alta Tecnología (CAT) de la UNAM. 